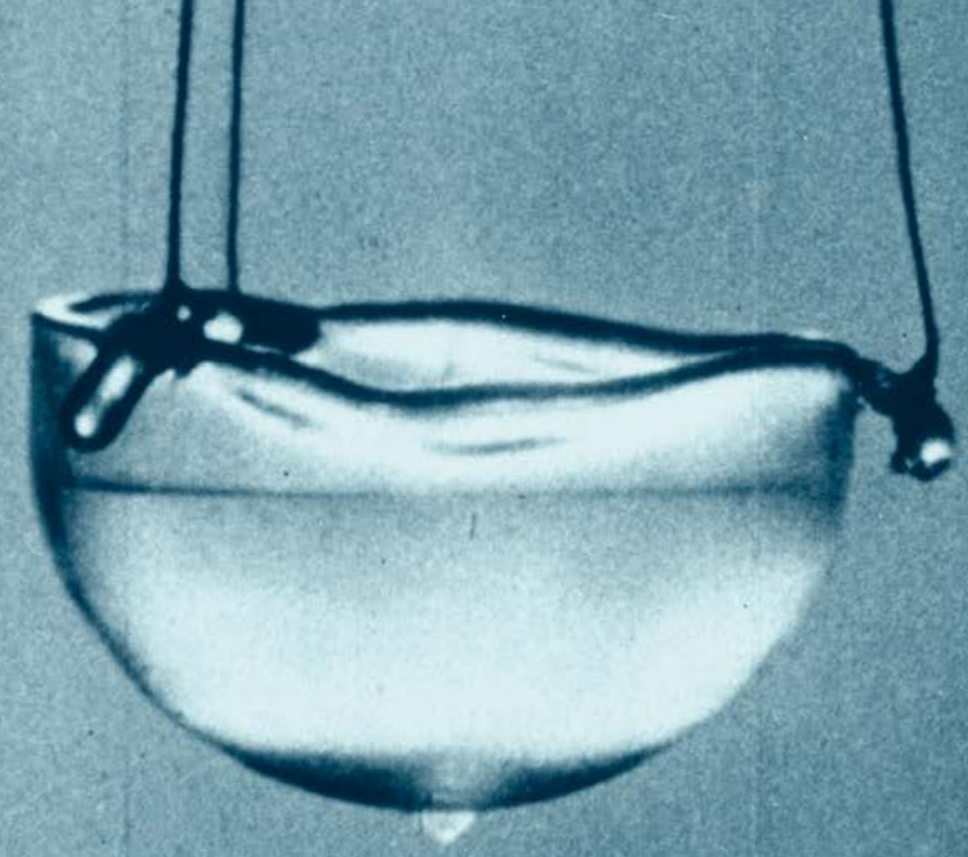

\title{
QUANTUM STORM IN A COLD CUP
}

Quantum turbulence, which manifests itself via a tangle of quantized vortices, occurs in quantum fluids, whose properties depend on quantum physics rather than classical physics. Here we report on two limiting forms of quantum turbulence which have been identified and how two-dimensional turbulence, until recently a mathematical idealization, has become experimental reality.

I Carlo F. Barenghi ${ }^{1}$ and Ladislav Skrbek ${ }^{2}$ - DOI: https://doi.org/10.1051/epn/2021305

I ' Joint Quantum Centre (JQC) Durham-Newcastle - School of Mathematics, Statistics and Physics - Newcastle University, Newcastle upon Tyne - NE1 7RU, United Kingdom

I ${ }^{2}$ Faculty of Mathematics and Physics - Charles University - Ke Karlovu 3, 121 16, Prague 2 - Czech Republic

$\mathrm{t}$ was the Renaissance genius Leonardo da Vinci, an acute observer of Nature, who first noticed that turbulence consists of eddies (or swirls, or vortices) of different sizes and strengths, which he sketched in a famous drawing. This insight was not followed up on until the late 1800's, when Osborne Reynolds reached the same conclusion and brought it to the attention of physicists.

Despite the great progress since the times of Reynolds, turbulence is still a challenge: it is a multi-scale nonlinear phenomenon in which billions of degrees of freedom are excited simultaneously and interact with each other. To complicate this statistical problem, the turbulent velocity field fluctuates wildly both in time and from point to point - it is essentially non-differentiable. Even the mathematical nature of the solutions of the governing equation of motion, the Navier-Stokes equation, has been questioned. In the year 2000, it was selected by the Clay Mathematics Institute as one the seven Millennium Problems whose solution is worth a prize of one million dollars each (to date, only one of the seven problems the Poincare conjecture - has been solved).

More recently, a new aspect of the turbulence problem has emerged: turbulence in quantum fluids, or quantum turbulence (QT) [1]. Key properties of quantum fluids depend on quantum physics rather than classical Newtonian physics, something which typically requires very low temperatures (lower than some critical temperature of the system). There are many quantum fluids, but here we $\bullet \bullet \bullet$ $\triangle$ A very cold cup of superfluid ${ }^{4} \mathrm{He}$, at temperature less than 2.2 Kelvin, where liquid ${ }^{4}$ He represents a quantum fluid of extraordinary physical properties that depend on quantum rather than classical physics. 
V FIG. 1:

Snapshot of a

small numerically computed tangle of vortex lines in ${ }^{4} \mathrm{He}$.

The vortex lines are represented as thick coloured tubes to visualize the 3D nature of QT (in reality, the vortex cores are about 10,000 times thinner than the average distance between the vortex lines). Note the helical deformations of the vortex lines (Kelvin waves) and the highly knotted topology of QT [2].
- consider ${ }^{4} \mathrm{He},{ }^{3} \mathrm{He}$ and atomic Bose-Einstein condensates (BECs) - small clouds of ultra-cold gases confined by magneto-optical traps at temperatures of order Kelvin, milliKelvin and microKelvin, respectively.

The first important property of these quantum fluids is superfluidity (or absence of viscosity, so named in analogy to superconductivity), which is the ability to flow, under some conditions, without any friction. Superfluidity is a consequence of the shape of the dispersion relation between energy and momentum of elementary excitations: unless an object moves faster than a critical velocity, it cannot lose energy to the fluid, which thus appears to the moving object as a background vacuum. The second important property is that in the zero temperature limit the entire fluid is described by a macroscopic complex wavefunction $\Psi$. At non-zero temperature there is also a thermal fraction (called the normal fluid in helium to distinguish it from the superfluid component) which behaves like an ordinary viscous fluid. The uniqueness of $\Psi$ and the basic rules of quantum mechanics imply that vorticity is constrained to thin cores of quantized vortices - vortex lines - of fixed strength: the quantum of circulation $\kappa=h / m$ where $h$ is the Planck constant and $m$ the mass of the relevant boson (such as the ${ }^{4} \mathrm{He}$ atom or a Cooper pair of ${ }^{3} \mathrm{He}$ atoms). The vortex axis, where $\Psi$ is suppressed, is a topological line defect (the phase is undefined), surrounded by a thin tubular region of depleted density, in the case of ${ }^{4} \mathrm{He}$ of tiny radius $a_{0} \approx 10^{-10} \mathrm{~m}$. This property is in sharp contrast to what happens in ordinary viscous fluids, where vorticity is unconstrained in both shape and strength.

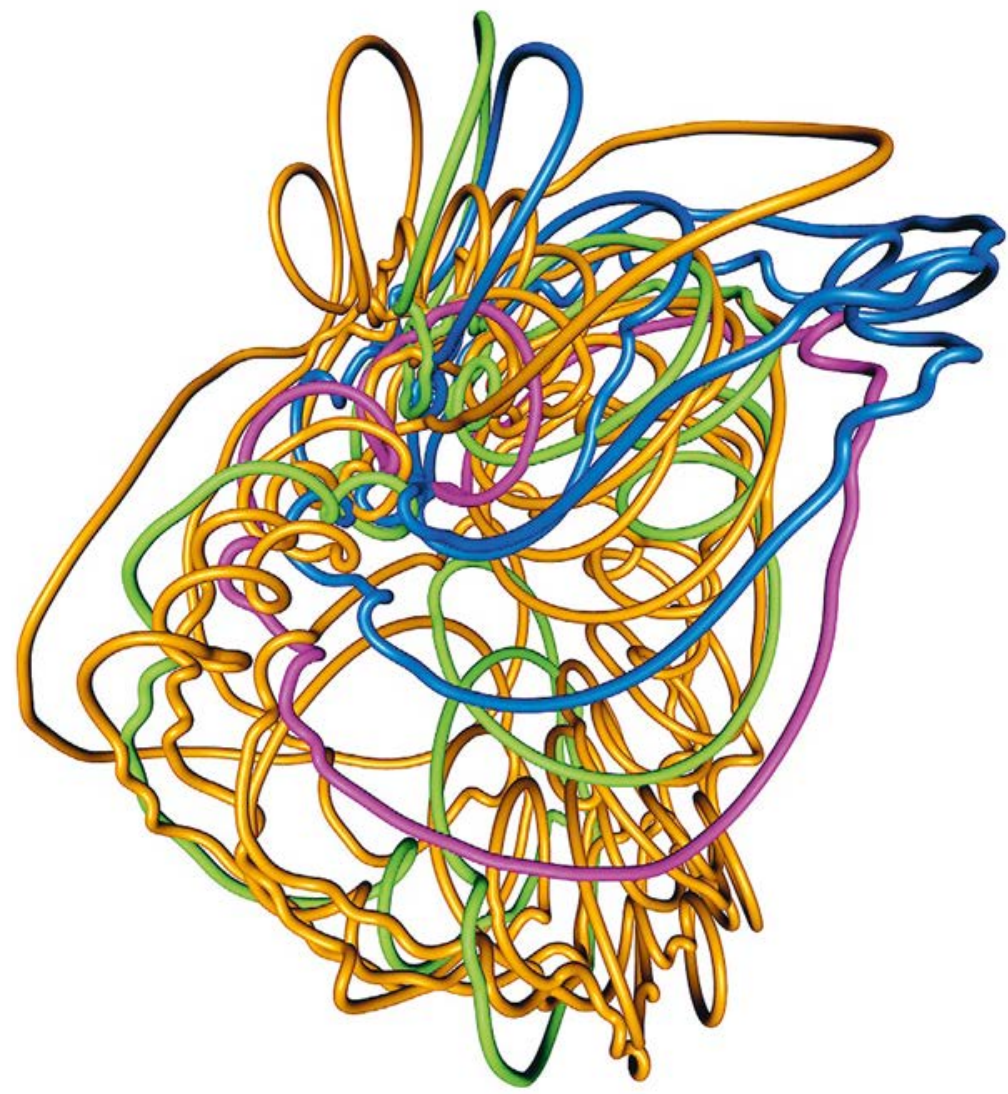

\section{Classical turbulence}

In order to capture the general features of ordinary (classical) three-dimensional (3D) turbulence in viscous fluids, physicists invented the concept of homogeneous isotropic turbulence (HIT), a state which is independent of position and orientation. An example of HIT is the turbulent flow inside a wind tunnel of width $D$ after subtracting the mean velocity. A statistically steady state of HIT is sustained by continuous energy input at rate $\varepsilon$ at some large scale $M<D$, where $D$ is the system size (for example, $M$ is the mesh size of the grid of the wind tunnel). This input compensates the losses $d E / d t=-\varepsilon$ of turbulent kinetic energy $E$ due to the fluid's kinematic viscosity, $v$. Nonlinearity ensures that the injected energy is shifted without losses to smaller and smaller length scales (a mechanism called the Richardson energy cascade) until it reaches a dissipation length scale $\eta$ (called the Kolmogorov length scale) which is small enough that kinetic energy is turned into heat. Simple dimensional analysis yields $\eta=(v / \varepsilon)^{1 / 4}$. An inertial range of scales develops between $M$ and $\eta$ where viscosity does not matter and the distribution of the kinetic energy among eddies is proportional to the $5 / 3$ power of the eddies' size (Kolmogorov's law), neglecting for simplicity fine details known as intermittency corrections.

\section{Quantum turbulence}

QT can be easily excited in superfluid ${ }^{4} \mathrm{He}$, in the B-phase of ${ }^{3} \mathrm{He}$ and in atomic BECs. Let us consider temperatures so low that the normal component is effectively absent. QT is then a disordered tangle of vortex lines (Fig. 1) which move each other and reconnect when they collide. Since there is no viscosity, there is no dissipation scale $\eta$. However, as an analogy with classical fluids, one can define a quantum length scale, $\ell_{\mathrm{Q}}$, by replacing $v$ with the quantum of circulation, $\kappa$ [3]. At scales larger than $\ell_{\mathrm{Q}}$, a small polarisation of vortex lines allows the stretching required to sustain the classical Richardson cascade. Indeed, at these scales experiments and numerical simulations display the same Kolmogorov distribution of kinetic energy among scales as observed in classical turbulence. At scales smaller than $\ell_{\mathrm{Q}}$ there is no polarisation and the quantisation of circulation prevents stretching individual vortex lines cannot stretch: at these scales, QT is unlike classical turbulence. Consistently, the velocity statistics are nearly Gaussian as in classical turbulence if probed at length scales larger than $\ell_{\mathrm{Q}}$ but become power-laws if probed at scales smaller than $\ell_{\mathrm{Q}}[4,5]$.

The classical Richardson energy cascade cannot proceed beyond $\ell_{\mathrm{Q}}$ but a non-linear transfer of energy further downscale is still possible along individual vortex lines. Fig. 1 shows that vortex lines sustain Kelvin waves, similar to those of a garden hose. The nonlinear interaction of Kelvin waves creates shorter and shorter waves in 
a second cascade process with no classical analogy called the Kelvin wave cascade, until kinetic energy is eventually dissipated, either by phonon emission (in ${ }^{4} \mathrm{He}$ ) or by exciting bound states in the vortex cores (in ${ }^{3} \mathrm{He}-\mathrm{B}$ ). In other words, in both classical and quantum turbulence, the final destiny of the energy input at large scale $M$ is the same: heat.

The emergence of the quantum scale in QT (its existence is a purely quantum effect, it vanishes in the quasi-classical limit of zero Planck constant), besides $M$ and $\eta$, adds a twist to the story. If $\ell_{\mathrm{Q}}$ is larger or similar to $\mathrm{M}$, the vortex lines remain at random with respect to each other without forming any partial polarization, hence there is neither Richardson cascade nor inertial range. This regime of QT, identified in superfluid helium, phase transitions and atomic BECs, is called Vinen turbulence; its fingerprint is the temporal decay of the vortex line length which behaves as $L \approx 1 / t$ for late times, unlike the $L \approx 1 / t^{3 / 2}$ of Kolmogorov turbulence [3].

At non-zero temperatures, quantum fluids behave as two-fluid systems consisting of an inviscid superfluid and a thermal viscous component which introduces friction on the motion of vortex lines. In atomic BECs the mean free path of the thermal component is large, while in ${ }^{4} \mathrm{He}$ above $1 \mathrm{~K}$ it is short enough to behave like a classical Navier-Stokes fluid (the normal fluid) which may easily become turbulent, too. This opens new scenarios and a rich variety of turbulent flow regimes which are being investigated. Moreover, in some quantum flows (such as in thermal counterflow of ${ }^{4} \mathrm{He}$, important for cryogenic engineering applications), QT can be simultaneously driven on large and small scales. A recent review [3] provides a general phenomenological understanding of rich variety of turbulent quantum flows of superfluid helium.

While the properties of helium are fixed by the temperature and pressure of an experiment, physical properties of atomic BECs (the sign and strength of the inter-atomic interaction, the density, shape, size and even the dimensionality of the system) can be tuned by the experimentalists. Unfortunately atomic condensates seldom contain more than $\approx 10^{6}$ atoms, hence they are relatively small: the ratio $D / a_{0}$, representing the number of linear degrees of freedom, is typically only $10-20$, which must be compared to the staggering $D / a_{0} \approx 10^{10}$ achieved with ${ }^{4} \mathrm{He}$. Despite the small size of atomic BECs, there is experimental evidence for the emergence of scaling laws [6]. Atomic BECs are thus ideal settings to study the crossover from chaos to turbulence.

\section{Two-dimensional quantum turbulence}

Atomic BECs that are so tightly confined in one direction that their thickness is of the order of $\mathrm{a}_{0}$ are essentially $2 \mathrm{D}$ systems. Given the same number of atoms, the ratio $\mathrm{D} / \mathrm{a}_{0}$ (hence the number of possible vortex lines in the systems) can be made much larger than in $3 \mathrm{D}$. BECs thus offer the opportunity of studying truly 2D turbulence, something which until recently was considered a mathematical idealization.

In $2 \mathrm{D}$ turbulence there is an inverse energy cascade from small to large-length scales (contrasting the 3D Richardson cascade in the other direction), hence the formation of large coherent vortex structures like Jupiter's Red Spot. In terms of discrete vortices, this effect is captured by Onsager's idea of the 2D vortex gas. In a confined system (such as an atomic BEC), the number of accessible states is not a monotonically increasing function of the energy, but reaches a maximum before decreasing. This property implies that at a high enough energy the temperature becomes formally negative; vortices of the same sign merge, creating large clusters of positive and negative vorticity, as already demonstrated in the laboratory [7].

\section{Conclusions}

Turbulence is a traditional interest of fluid dynamicists, engineers, geophysicists and astrophysicists. This is changing. Novel forms of turbulent quantum flows are being explored, emerging in helium superfluids, single and two-component atomic condensates, polaritons, spinor condensates, quantum ferrofluids and models of the early Universe. Quantum turbulence, demonstrating the universality of concepts such as energy cascades and reconnections, represents a fast-growing branch of physics.

\section{About the authors

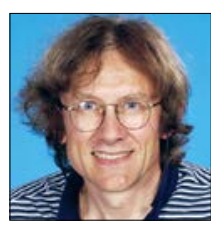 \\ Carlo F. Barenghi is a Professor of Physics at School of Mathematics, Statistics and Physics, Newcastle University, Newcastle upon Tyne, NE1 7RU, United Kingdom.

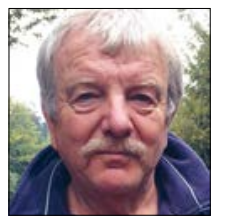 \\ Ladislav Skrbek is a Professor of Physics at School of Physics, Faculty of Mathematics and Physics, Charles University, 12116 Prague, Czech Republic.}

\section{References}

[1] C. F. Barenghi, L. Skrbek and K. R. Sreenivasan, Proc. Nat. Acad. Sci. USA 111, 4647 (2014)

[2] R. G. Cooper, M. Mesgarnezhad, A.W. Baggaley and C. F. Barenghi, Scientific Reports 9, 10545 (2019)

[3] L. Skrbek, D. Schmoranzer, S. Midlik and K. R. Sreenivasan, Proc. Nat. Acad. Sci. USA 118, e2018406118 (2021)

[4] A.W. Baggaley and C.F. Barenghi, Phys. Rev. E 84, 067301 (2011)

[5] M. LaMantia and L. Skrbek, Europhys. Lett. 105, 46002 (2014).

[6] N. Navon, A. L. Gaunt, R. P. Smith, and Z. Hadzibabic, Nature 539, 72 (2016)

[7] S. P. Johnstone, A. J. Groszek, P. T. Starkey, C. J. Billington, T. P. Simula and K. Helmerson, Science 364, 1267 (2019) 